Citation: K. Kanta Roy, Md MuzahidE-Rahman, Kishowar-E-Mustarin, Md M. A. Reza, P. K. Malaker, N. C. Deb Barma, X. He, P. K. Singh (2021) First report of blast of durum wheat in Bangladesh, caused by Magnaporthe oryzae pathotype Triticum. Phytopathologia Mediterranea 60(1): 105-111. doi: 10.36253/phyto-11821

Accepted: November 3, 2020

Published: May 15, 2021

Copyright: () 2021 K. Kanta Roy, Md Muzahid-E-Rahman, Kishowar-E-Mustarin, Md M. A. Reza, P. K. Malaker, N. C. Deb Barma, X. He, P. K. Singh. This is an open access, peer-reviewed article published by Firenze University Press (http://www.fupress.com/pm) and distributed under the terms of the Creative Commons Attribution License, which permits unrestricted use, distribution, and reproduction in any medium, provided the original author and source are credited.

Data Availability Statement: All relevant data are within the paper and its Supporting Information files.

Competing Interests: The Author(s) declare(s) no conflict of interest.

Editor: Andy Tekauz, Cereal Research Centre, Winnipeg, MB, Canada.
New or Unusual Disease Reports

\section{First report of blast of durum wheat in Bangladesh, caused by Magnaporthe oryzae pathotype Triticum}

\author{
Krishna Kanta ROY ${ }^{1, \star}$, Md MUZAHID-E-RAHMAN ${ }^{1}$, KISHOWAR-E- \\ MUSTARIN $^{1}$, Md Mostofa Ali ReZA ${ }^{1}$, Paritosh Kumar MALAKER ${ }^{1}$, \\ Naresh Chandra DEB BARMA ${ }^{1}$, Xinyao HE ${ }^{2}$, Pawan Kumar SINGH ${ }^{2, *}$ \\ ${ }^{1}$ Bangladesh Wheat and Maize Research Institute (BWMRI), Nashipur, Dinajpur-5200, \\ Bangladesh \\ ${ }^{2}$ International Maize and Wheat Improvement Centre (CIMMYT), El Batan, Texcoco, \\ Mexico \\ ^Corresponding authors. E-mail: rkrishnaroy666@gmail.com; pk.singh@cgiar.org
}

\begin{abstract}
Summary. Durum wheat (Triticum turgidum var. durum Desf.) is an important cereal crop in many regions of the world. In March of 2018 and 2019, symptoms typical of blast were frequently observed on durum wheat plants under field conditions in Jashore, Bangladesh. The putative causal pathogen was isolated from infected wheat spike specimens onto potato dextrose agar and oatmeal agar, and was identified from mono-conidium cultures as Magnaporthe oryzae, based on morphological features. The pathotype of the fungus was identified as Triticum, based on comparative molecular analyses of ITS sequences and MoT3 specific markers. BLAST analysis revealed $>99.8 \%$ similarity with $M$. oryzae/P. oryzae, retrieved from the NCBI Genebank. This was confirmed through amplification of the predicted products with MoT3 primers in PCR analysis. Pathogenicity was confirmed by inoculating healthy durum wheat seedling leaves and spikes with a conidium suspensions of $M$. oryzae isolate DuBWMRI1901.2A. The fungus produced similar symptoms on inoculated leaves and spikes as those observed in the field, and was subsequently re-isolated, fulfilling Koch's postulates. This is the first report of blast of durum wheat caused by Magnaporthe oryzae pathotype Triticum in Bangladesh.
\end{abstract}

Keywords. Isolation, morphological detection, molecular analysis, pathogenicity.

\section{INTRODUCTION}

Durum wheat (Triticum turgidum var. durum) is the second most cultivated species of wheat after bread wheat, accounting for 5 to $8 \%$ of global wheat production, and is the $10^{\text {th }}$ most important cereal crop overall (Kabbaj et al., 2017). Durum wheat is cultivated under diverse climatic conditions in West Asia and North Africa (WANA) and is the most sustainable wheat type in the Mediterranean basin due to its tolerance to hot and dry environmental conditions. It remains a significant staple food crop for mar- 
ginalized farmers due to its potential for large-scale production and high monetary returns (Yahyaoui et al., 2000; Sall et al., 2019). The largest durum wheat producer is the European Union, with projected production in 2020 of 309 million bushels, followed by Canada (162), Turkey (132), Algeria (118), U.S.A. (59) and Morocco (48) (NDWC, 2020). The high protein grain content and hard structure make durum wheat ideal for producing pasta products, and it is therefore often referred as 'pasta wheat'. In addition, it can also be used to make bulgur, couscous, freekeh, puffed cereals, desserts, filler for pastries, and unleavened breads. In Bangladesh, wheat crops are only grown during winter. Although durum wheat is a non-traditional minor cereal in Bangladesh, it still has significant commercial importance (BARI, 2015). The Bangladesh Wheat and Maize Research Institute (BWM$\mathrm{RI})$ produces durum grain on a limited field scale $(<0.2$ ha) to explore its production and marketing potential in Bangladesh. Despite its current limited production, durum wheat may have good prospects in future as a niche market crop in the country.

Durum wheat can be severely affected by several pests and diseases. The most important diseases include stripe rust, Septoria tritici blotch, Fusarium head blight, stem rust, leaf rust, tan spot, root rot, crown rot, and common bunt (Yahyaoui et al., 2000; Haile et al., 2019). Under favourable environmental conditions yield losses in the WANA region due to diseases can reach $40 \%$ (Yahyaoui et al., 2000). In March of 2018 and 2019, a disease with symptoms similar to those of wheat blast, previously identified on bread wheat in Bangladesh (Malaker et al., 2016), was observed on durum wheat for the first time in experimental field plots in Jashore, Bangladesh. Blast is caused by the hemi-biotrophic ascomycete fungus Magnaporthe oryzae (Catt.) B. C. Couch 2002 (anamorph Pyricularia oryzae Cavara 1892) (Couch and Kohn 2002; Couch et al., 2005; Zhang et al., 2016). There was no record of blast on durum wheat under field conditions in Bangladesh prior to this report. Magnaporthe oryzae is versatile in nature, having a group of sub-species, and can attack $50+$ species in the 'Poaceae', including wheat, rice, triticale, barley, millet, oat, and others (Sundaram et al., 1972; Ou, 1985; Igarashi et al., 1986; Urashima et al., 1993; Kato et al., 2000; Urashima et al., 2004; Roy et al., 2020). The pathogen is host-specific: the Triticum pathotype is responsible for infecting wheat, the Oryza pathotype, rice, the Setaria pathotype, millet, the Lolium pathotype, annual and perennial ryegrass, among others. The first epidemic of the Triticum pathotype occurred in Parana, Brazil in 1985 (Igarashi et al., 1986). The initial outbreak outside of South America was observed in wheat in Bangladesh in Febru- ary 2016 (Malaker et al., 2016), devastating the season's harvest. This pathotype can also attack other barley and triticale, as observed in Bangladesh in 2019 and confirmed by morphological and molecular analyses (Roy et al., 2020; Roy, unpublished). Positive cross-infection of MoT isolates on wheat, triticale, durum, and barley was observed under laboratory conditions at BWMRI (Roy, unpublished).

In the present investigation, symptoms of blast were observed in durum wheat under field conditions. Hence, the aim of the present study was to identify the causative pathogen by confirming morphological features, conducting molecular analyses using ITS sequences and pathotype-specific markers, and conducting pathogenicity tests.

\section{MATERIALS AND METHODS}

\section{Isolation, purification, and detection of the pathogen}

To isolate the disease-causing pathogen, four to five diseased spikes were randomly collected from blastaffected plots in Jashore, Bangladesh in 2018, and brought to the BWMRI pathology laboratory. The infected spikes were partially or fully bleached with shriveled grains, indicating pathogen infections at an early stage of grain development. Special care was taken to ensure samples were fresh and therefore less likely to be contaminated with other fungi. Small portions $\left(<1 \mathrm{~cm}^{2}\right)$ of symptomatic spike tissues were surface disinfected in $1 \%$ Clorox $(\mathrm{NaOCl})$ solution for $20-30 \mathrm{sec}$, and were then rinsed twice with sterile distilled water. The samples were incubated in darkness at $25-28^{\circ} \mathrm{C}$ on threelayer moistened Whatman blotter paper to promote fungal sporulation. After $24 \mathrm{~h}$, conidiophores and conidia developed on the tissue samples. Single conidia were transferred onto Petri dishes containing potato dextrose agar (PDA) (200 g sliced potato, $15 \mathrm{~g}$ agar powder, and $12 \mathrm{~g}$ dextrose in $1000 \mathrm{~mL}$ distilled water). These were incubated for 5-7 d in a laminar air flow chamber with continuous fluorescent light at $28 \pm 2{ }^{\circ} \mathrm{C}$ and $40-42 \%$ relative humidity to produce single-conidium isolate cultures. The morphology of two isolates was recorded, including colony characteristics and conidium colour, shape, and size.

\section{Molecular analyses with ITS sequences and MoT3 primers}

The pathotype of the two isolates was confirmed through molecular analyses using ITS sequencing (White et al., 1990) and PCR amplification with MoT3 
primer sets (Pieck et al., 2017). To confirm the pathotype of two of the isolates through ITS sequencing, total genomic DNA was extracted from mycelia of the isolates separately using a Wizard Genomic DNA Purification Kit (Promega) following the manufacturer's protocol. Molecular confirmation was first performed using the internal transcribed spacer (ITS) region of the ribosomal DNA by amplifying the isolates with ITS5 (GGAAGTAAAAGTCGTAACAAGG) and ITS4 (TCCTCCGCTTATTGATATGC) primers. The primers were synthesized by First BASE Laboratories (Malaysia), and the resulting products were sequenced and aligned using BioEdit 7.2. The sequences were submitted to GenBank under the following accession numbers: LC554422 (isolate DuBWMRI1901.2A) and LC554423 (isolate DuBWMRI1902.76C), and were analyzed using BLAST (https: //blast.ncbi.nlm.nih.gov/Blast). A phylogenetic tree was constructed with the neighbour-joining likelihood method with 1000 bootstrap replications using the MEGA-X model (Kumar et al., 2018). To confirm the pathotype with MoT3 primer sets (MoT3 primers are responsible for the identification of wheat blast fungi), the protocol described above was used to extract genomic DNA from the isolates. PCR amplification was performed on the samples in $100 \mu \mathrm{L}$ PCR tubes using the GoTaq G2 Green Master Mix (Promega) and the MoT3 specific primers- MoT3F (GTCGTCATCAACGTGACCAG) and MoT3R (ACTTGACCCAAGCCTCGAAT). PCR was performed as described by Pieck et al. (2017) using a Thermal Cycler (Veriti, Applied Biosystems). The PCR cycling conditions were as follows: initial denaturation occurred at $94^{\circ} \mathrm{C}$ for $3 \mathrm{~min}$, followed by 30 cycles of denaturation at $94^{\circ} \mathrm{C}$ for $1 \mathrm{~min}$, annealing at $62^{\circ} \mathrm{C}$ for $2 \mathrm{~min}$, extension at $72^{\circ} \mathrm{C}$ for $1 \mathrm{~min} 30 \mathrm{sec}$, and final elongation at $72^{\circ} \mathrm{C}$ for $10 \mathrm{~min}$. Five microliters of each amplification mixture were verified by agarose $(1 \% \mathrm{w} / \mathrm{v})$ gel electrophoresis in $0.5 \times$ Tris-borate-EDTA (TBE) buffer. The presence or absence of the $361 \mathrm{bp}$ MoT-specific PCR band was observed after electrophoresis. To test the specificity of MoT3 primers, several other blast-causing isolates from wheat (positive), rice, triticale, barley, and foxtail millet were included, and water was used as a negative control.

\section{Pathogenicity tests on spikes and seedling leaves}

Pathogenicity tests were carried out using seedling leaves and spikes of durum wheat genotype BDW 08 . The seedlings were grown in $4 \times 3 \times 2.5 \mathrm{~cm}$ plastic pots, and adult plants in $15 \times 13 \times 7 \mathrm{~cm}$ pots, under laboratory conditions. There were four pots each for seedlings and for adult plants, with ten seedlings or adult plants in each pot. Seedlings at the two-leaf stage (12-14 d after sowing) and spikes (2-3 d after initiation of heading) were inoculated with isolate DuBWMRI1901.2A. To prepare conidia for MoT inoculation, inocula were multiplied on oatmeal agar (OMA) (50 g oatmeal and 15 g agar powder in $1000 \mathrm{~mL}$ distilled water). Sections of PDA-cultured MoT fungus growth were transferred onto $\mathrm{OMA}$, and were then incubated at $27-29^{\circ} \mathrm{C}$ and $40-45 \%$ relative humidity for $7-10 \mathrm{~d}$ to promote conidium production. and then harvested. A conidium suspension was then prepared by scraping the conidia from OMA plates into a $100 \mathrm{~mL}$ capacity beaker using a camel hair brush. The suspension was filtered through two layers of cheesecloth, and two drops of $0.01 \%$ Tween 20 solution were added to $100 \mathrm{~mL}$ of aqueous conidium suspension. The suspension was stirred gently for $10 \mathrm{~min}$ to thoroughly disperse the conidia. The test seedlings and adult (headed) plants were inoculated using the suspension of 30,000 conidia $\mathrm{mL}^{-1}$ using a hand sprayer. The suspension was applied onto plant leaves and spikes to run-off. Two series of inoculations using seedling leaves and adult-plant spikes were performed using the same procedure. Some leaves and spikes were sprayed with water only to serve as negative controls. The inoculated leaves were incubated in continuous darkness for 36 $\mathrm{h}$ and spikes for $48 \mathrm{~h}$, at $27 \pm 2^{\circ} \mathrm{C}$ and 80 to $85 \%$ relative humidity (RH) in a 'polyhood' chamber (a metallic framework covered with a transparent polyethylene sheet). Following incubation, all pots were removed from the chamber and placed onto a laboratory bench for symptom expression.

\section{RESULTS AND DISCUSSION}

Putative blast symptoms were observed in three durum wheat plots, one of $6 \mathrm{~m}^{2}$ in 2018, and two of $1 \mathrm{~m}^{2}$ in 2019. In these plots, $30-50 \%$ of the total spikes were infected. Symptoms were found primarily on spikes, but also on leaves and stems. Spike symptoms were partially bleached spikes typical of blast (Figure $1, A_{1}$ and $A_{2}$ ). These had visible dark gray or brown infection points on each rachis, and sometimes exhibited gray to white fungus sporulation (Figure $1 \mathrm{D}$ ). Infection of heads at the initial stage of anthesis led to aborted or shriveled and distorted grains with low test weights. Leaves with blasted symptoms had typical eye-shaped or elongated lesions with white centres and brown margins (Figure 1 B). Foliar symptoms were mostly observed on old leaves and rarely on young or flag leaves. Elongated lesions were also observed on stems; these had white centres and brown or black margins (Figure 1 B). They were 

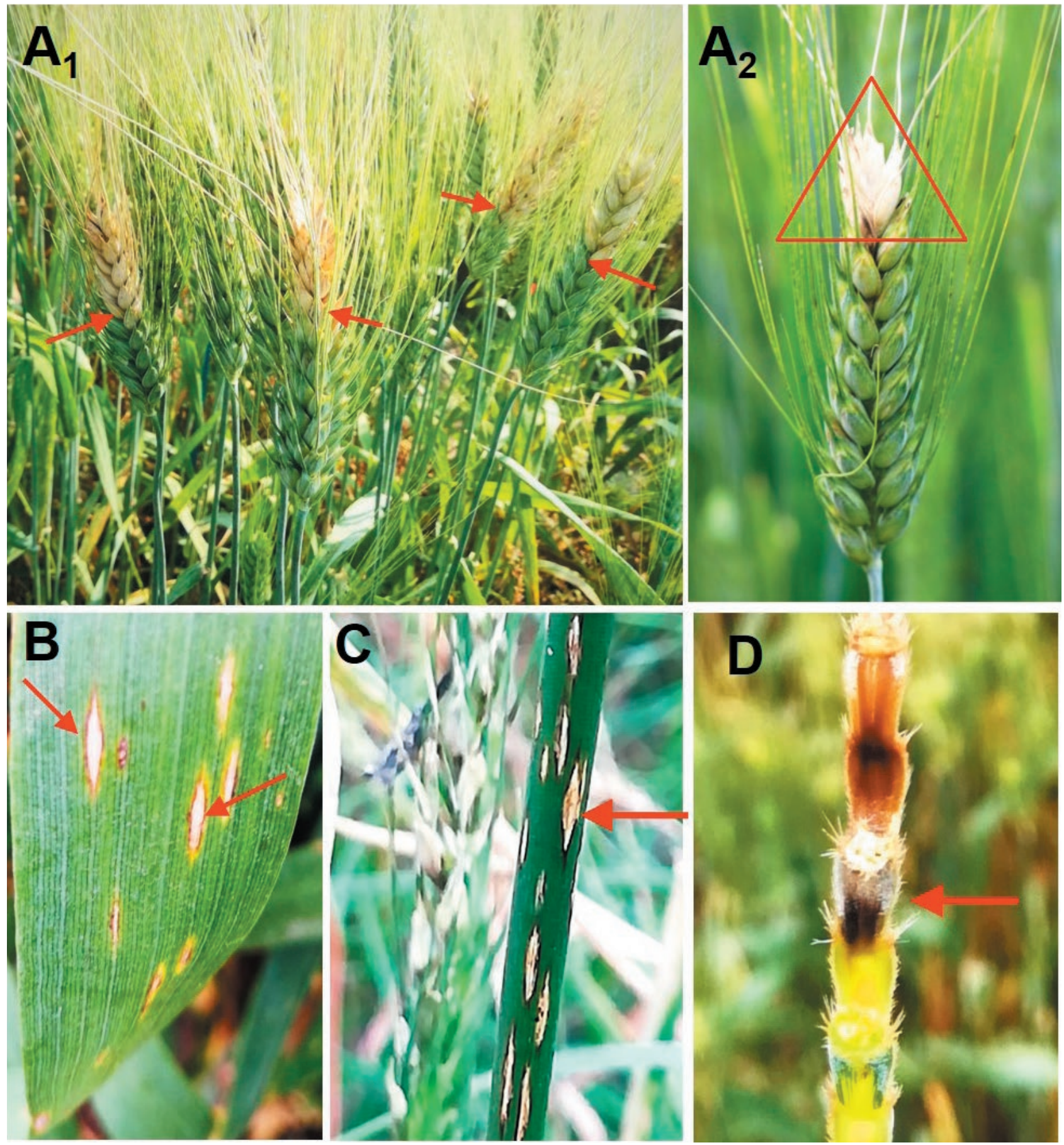

Figure 1. Field symptoms of durum wheat blast on different plant parts: $\left(A_{1}\right.$ and $\left.A_{2}\right)$ typical partially-bleached spikes; (B) elongated or eyeshaped lesions on durum leaf with white centers surrounded by brown margins; (C) elongated lesion on stem with white center and dark brown or black margins; and (D) dark gray or brown infection points and gray to white sporulation in rachis.

observed rarely, and may have only developed when conditions particularly favoured disease development.

Microscope examination of affected samples showed vegetative and reproductive growth of the pathogen.
Fungus growth was observed on rachis surfaces and on foliar lesions under a light microscope after $24-36 \mathrm{~h}$ of incubation (Figure 2, A and B). Young conidia of both isolates were pyriform, two-septate (three-celled), and 
translucent; over time these became grayish or slightly darkened (Figure $2 \mathrm{G}$ ), and sometimes appeared to be single-celled. Conidia were of uniform shape, although their sizes varied from $16-25 \times 7-10 \mu \mathrm{m}$. The middle cell of each conidium was wider and darker than the terminal cells, and each conidium tapered towards its apex. When conidia were water-mounted on glass slides, germtubes were evident within 2-3 h. Conidiophores were observed as coloured, erect, and cylindrical, with darkened basal portions and apical conidia. Conidiophores each bore seven to nine conidia acrogenously.

Colonies of the two isolates on PDA were gray to dark gray after 7 to $10 \mathrm{~d}$ incubation (Figure 2, C and E). Colonies of isolate DuBWMRI1901.2A were darker than those of DuBWMRI1902.76C. Puffy mycelial growth and a single concentric ring were observed in both isolates. On OMA, profuse grayish to white conidium masses were found after 8-12 d incubation (Figure 2, D and F). In the laminar flowhood, optimum conditions for conidium production were $28-29^{\circ} \mathrm{C}$ and $40-45 \%$, and continuous florescent light. Conidium production on rachis tissues was more profuse than on leaf tissues.

The morphological features of the conidia were identical to Magnaporthe oryzae as described by Subramanian (1968). Both isolates were placed in long-term storage on dried filter paper at $-81^{\circ} \mathrm{C}$ at the BWMRI pathology laboratory for future use. These can be accessed universally.

The pathotype of the isolated M. oryzae was confirmed by molecular analyses of the two representative isolates, DuBWMRI1901.2A and DuBWMRI1902.76C, using the internal transcribed spacer (ITS) region primer

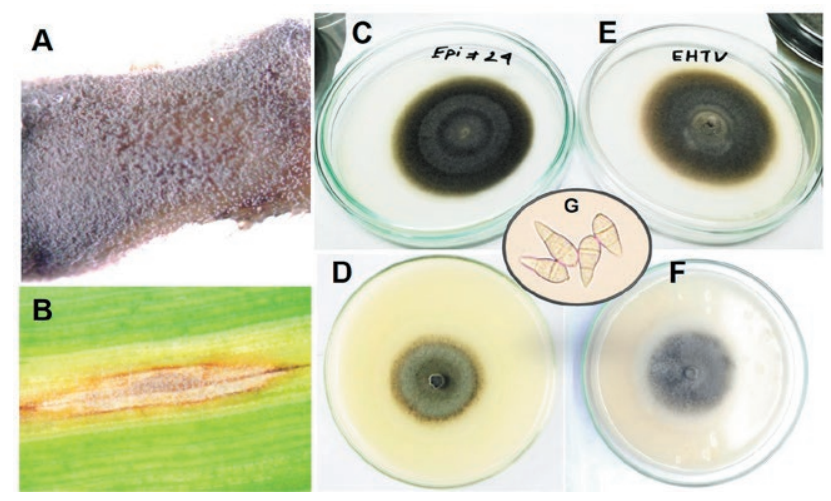

Figure 2. Growth of durum blast pathogen isolates on infected rachis and leaf tissues, and in PDA and OMA cultures after incubation under laboratory conditions. (A) gray to dark gray conidia of the fungus on an incubated rachis; (B) grayish conidia spores on an incubated leaf lesion; mono-conidium colonies of isolate DuBWMRI1901.2A on PDA (C), and OMA (D), and of DuBWMRI1902.76C PDA (E) and OMA (F); and (G) compound light microscope image of two-septate pyriform conidia.

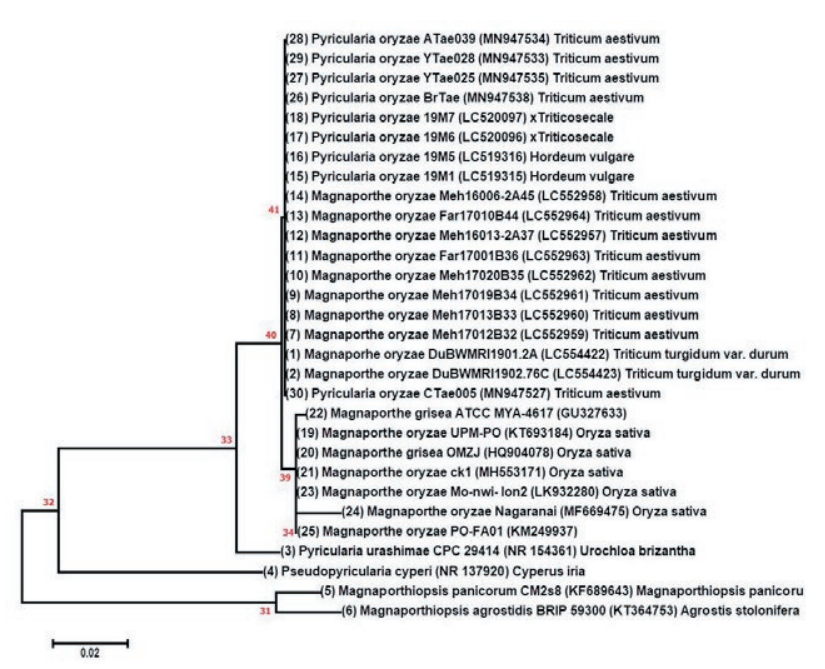

Figure 3. Neighbour-joining phylogenetic tree of Magnaporthe oryzae isolates DuBWMRI1901.2A and DuBWMRI1902.76C with 28 related rDNA-ITS sequences from GenBank. Numbers at the nodes indicate bootstrap values generated from 1000 replicates, and the scale bar indicates the number of nucleotide substitutions.

sets and the pathotype-specific MoT3 primers. The ITS sequences of the isolates were $>99.8 \%$ similar to several M. oryzae/P. oryzae sequences available in GenBank. The phylogenetic tree (Figure 3) grouped the Bangladeshi durum blast isolates together with other $M$. oryzae/P. oryzae isolates from Triticum aestivum, $\times$ Triticosecale and Hordeum vulgare from Paraguay and Bangladesh. Based on the aligned sequences of ITS, the constructed neighbour-joining likelihood phylogenetic tree confirmed that the two blast isolates were identical to Magnaporthe oryzae (Subramanian 1968). Similarly, MoT3 primer results demonstrated that the two blast isolates were amplified with the band ( $361 \mathrm{bp}$ ) identical to those of wheat, triticale and barley blast isolates during PCR assay, while there was no PCR amplification found with rice and foxtail millet blast isolates (Figure 4).

The pathogenicity tests of the $M$. oryzae isolates gave at $48-72 \mathrm{~h}$ post-inoculation, eye-shaped water-soaked lesions developed on the seedling leaf blades (Figure 5 A), while there were no symptoms observed on watertreated plants (controls). The lesions had gray centres and dark green margins. The lesions became elongated and developed grayish centres with brown margins (Figure $5 \mathrm{~B}$ ); ultimately whole leaves became blighted. In contrast, the inoculated spikes were only partially bleached 13-15 d after inoculation (Figure $5 \mathrm{C}$ ). The water-inoculated spikes remained symptomless. Magnaporthe oryzae was re-isolated on PDA plates (Figure 5 E) from the symptomatic leaves of inoculated plants, which produced profuse conidia within $24 \mathrm{~h}$ of incubation 


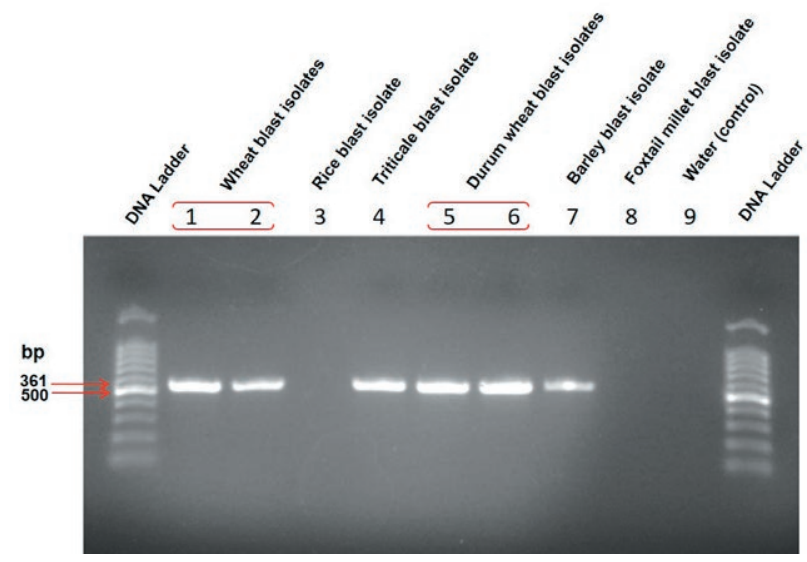

Figure 4. $P C R$ amplification of durum wheat blast isolates DuBWMRI1901.2A (lane 5) and DuBWMRI1902.76C (lane 6), and blast isolates from wheat (lanes 1 and 2), rice (lane 3), triticale (lane 4), barley (lanes 7), and foxtail millet (lane 8), using MoT3 primer sets for confirming the Triticum pathotype of the Magnaporthe ory$z a e$ pathogen, during molecular detection assay.

(Figure $5 \mathrm{D}$ ). Conidia of the re-isolated pathogen were identical to those used for inoculations, and thus fulfilling Koch's postulates for the inoculated fungi (Cohen 1994).
Based on the morphological features of the selected isolates (conidium shape, size and colour), molecular analyses (ITS sequences and MoT3 markers) and pathogenicity tests, the causal organism of durum wheat blast observed in field plots was confirmed to be Magnaporthe oryzae pathotype Triticum. This is the first report of blast of durum wheat in Bangladesh, by Magnaporthe oryzae pathotype Triticum.

\section{ACKNOWLEDGEMENTS}

This study was financially supported by grants from the Australian Centre for International Agricultural Research (ACIAR), and CRP WHEAT and Krishi Gobeshona Foundation (KGF), Bangladesh.

\section{LITERATURE CITED}

BARI, 2015. Bangladesh Agricultural Research Institute, Joydebpur, Dhaka. Annual Report (2014-2015), 7 p.

Cohen J., 1994. Fulfilling Koch's postulates. Science 266: 1647.

Couch B.C., Kohn L.M., 2002. A multilocus gene genealogy concordant with host preference indicates segre-

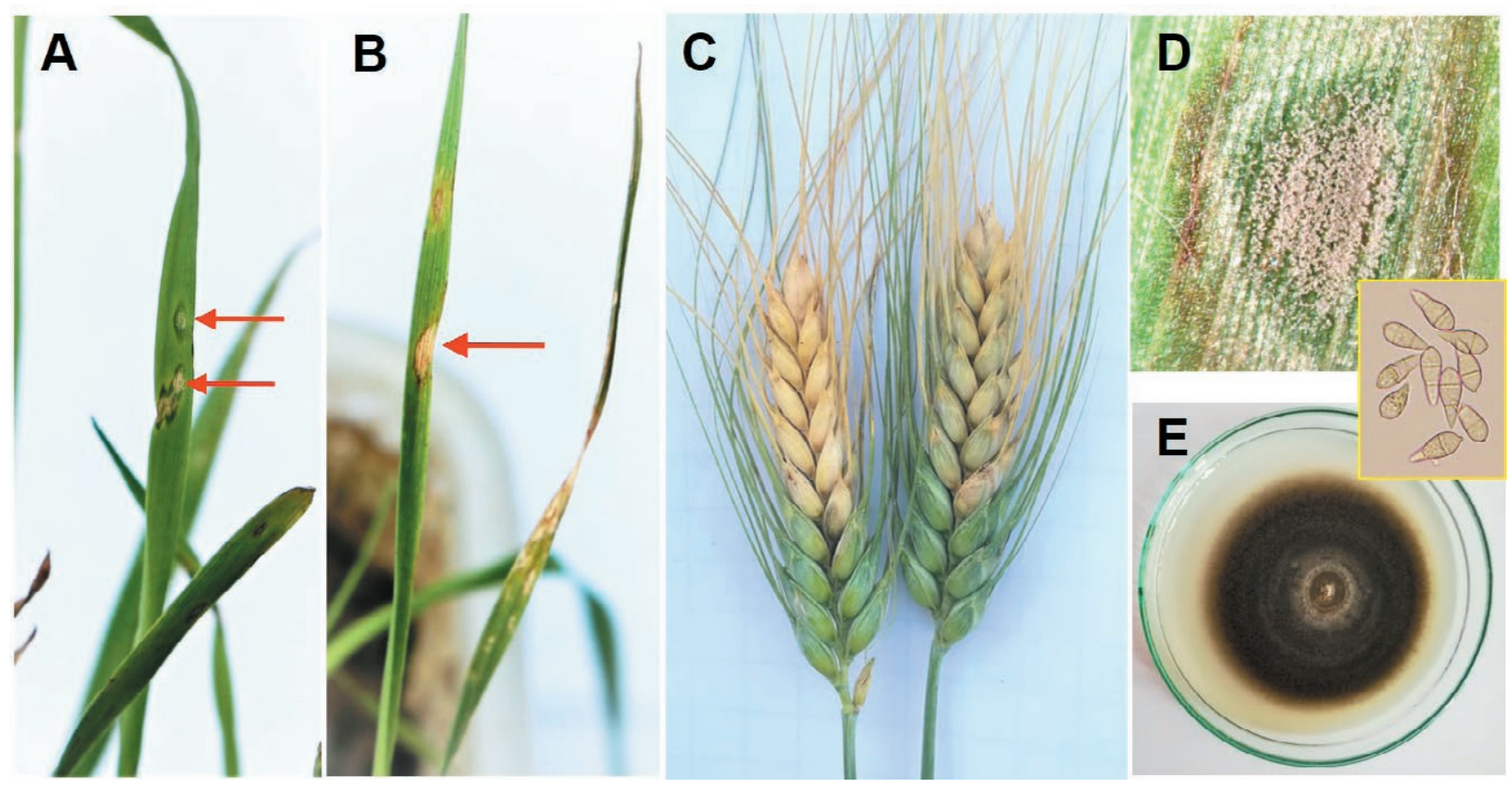

Figure 5. Results from pathogenicity tests of the Magnaporthe oryzae putative pathogen used to fulfill Koch's postulates. (A) typical eyeshaped water-soaked lesions on seedling leaves 48-72 h after inoculation with isolate DuBWMRI1901.2A; (B) elongated lesion with a grayish centre and brown margins 5-7 d post-inoculation; (C) partially bleached spikes 12-14 d post-inoculation; (D) gray conidium growth on a detached leaf lesion after $24 \mathrm{~h}$ incubation on moist blotter paper; and (E) re-isolated inoculated isolate, and conidia (inset). 
gation of a new species, Magnaporthe oryzae, from M. grisea. Mycologia 94: 683-693.

Couch B., Fudal I., Lebrun M., Tharreau D., Valent B., ... Kohn L., 2005. Origins of host-specific populations of the blast pathogen Magnaporthe oryzae in crop domestication with subsequent expansion of pandemic clones on rice and weeds of rice. Genetics 170: 613-630.

Haile J.K., N’Diaye A., Walkowiak S., Nilsen K.T., Clarke J.M., ... Pozniak C.J., 2019. Fusarium Head Blight in Durum Wheat: Recent Status, Breeding Directions, and Future Research Prospects. Phytopathology 109 (10): 1664-1675.

Igarashi S., Utiamada C.M., Igarashi L.C., Kazuma A.H., Lopes R.S., 1986. Occurrence of Pyricularia sp. in wheat (Triticum aestivum L.) in the State of Parana, Brazil. Fitopatologia Brasileira 11: 351-52.

Kabbaj H., Sall A.T., Al-Abdallat A., Geleta M., Amri A., ... Bassi F.M., 2017. Genetic diversity within a global panel of durum wheat (Triticum durum) landraces and modern germplasm reveals the history of alleles exchange. Frontiers in Plant Science 8: 1277.

Kato H., Yamamoto M., Yamaguchi-Ozaki T., Kadouchi H., Iwamoto Y., ... Naoki M.O.R.I., 2000. Pathogenicity, mating ability and DNA restriction fragment length polymorphisms of Pyricularia populations isolated from Gramineae, Bambusideae and Zingiberaceae plants. Journal of General Plant Pathology 66: 30-47.

Kumar S., Stecher G., Li M., Knyaz C., Tamura K., 2018. MEGA X: Molecular evolutionary genetics analysis across computing platforms. Molecular Biology and Evolution 35(6): 1547-1549.

Malaker P.K., Barma N.C.D., Tiwari T.P., Collis W.J., Duveiller. E., ... Valent B., 2016. First report of wheat blast caused by Magnaporthe oryzae pathotype triticum in Bangladesh. Plant Disease 100(11): 2330.

NDWC, 2020. World Durum Production. (Source: International Grains Commission Xldata/world/all wheat/ wheat/prod). Available at: https: //www.ndwheat. com/uploads/6/world-web-charts

Ou S.H., 1985. Blast. In: Ou, S. H. (ed.). Rice Diseases, $2^{\text {nd }}$ edition. CABI, Wallingford, UK, 109-201 pp.

Pieck M.L., Ruck A., Farman M.L., Peterson G.L., Stack J.P., ... Pedley K.F., 2017. Genomics based marker discovery and diagnostic assay development for wheat blast. Plant Disease 101: 103-109.

Roy K. K., Rahman M.M.E., Reza M.M.A., Mustarin K.E., Malaker P.K., ... Singh P.K., 2020. First report of triticale blast caused by the fungus Magnaporthe oryzae pathotype Triticum in Bangladesh. Canadian Journal of Plant Pathology (DOI: 10.1080/07060661.2020.1793223).
Sall A.T., Chiari T., Legesse W., Seid-Ahmed K., Ortiz R., ... Bassi F.M., 2019. Durum wheat (Triticum durum Desf.): origin, cultivation, and potential expansion in Sub-Saharan Africa. Agronomy 9: 263.

Subramanian C.V., 1968. Pyricularia oryzae. CMI Descriptions of Pathogenic Fungi and Bacteria.

Sundaram N.V., Palmer L.T., Nagarajan K., Prescott J.M., 1972. Disease survey of sorghum and millet in India. Plant Disease 56: 740-743.

Urashima A.S., Igarashi S., Kato H., 1993. Host range, mating type and fertility of Pyricularia grisea from wheat in Brazil. Plant Disease 77: 1211-1216.

Urashima A.S., Lavorent N.A., Goulart A.C.P., Mehta Y.R., 2004. Resistance spectra of wheat cultivars and virulence diversity of Magnaporthe grisea isolates in Brazil. Fitopatologia Brasileira 29: 511.

White T.J., Bruns T., Lee S., Taylor J., 1990. Amplification and direct sequencing of fungal ribosomal RNA genes for phylogenetics. A Guide to Methods and Applications. Academic Press, San Diego, 315-322 pp.

Yahyaoui A., Hakim S., Al-Naimi M., Nachit M.M., 2000. Multiple disease resistance in durum wheat (Triticum turgidum L. var. durum). In: Royo C., Nachit M., DiFonzo N., and Araus J. L. (eds.). Durum wheat improvement in the Mediterranean region: New challenges. CIHEAM, Zaragoza, Spain, 387-392 pp.

Zhang N., Luo J., Rossman A.Y., Aoki T., Chuma I., ... Xu J.R., 2016. Generic names in Magnaporthales. IMA Fungus 7: 155-159. 